\title{
Rapport des réunions de l’EAP
}

Lisbonne, 17-19 octobre 2019

Peter Schulthess

Les réunions de la European Association for Psychotherapy (EAP) se sont déroulées relativement dans le calme cette fois-ci. L'EAP est consciente qu'il y aura des changements dans le paysage psychothérapeutique européen dans les années à venir.

La nouvelle loi sur les psychothérapeutes en Allemagne aura un impact important, d'autant plus qu'elle améliorera également la rémunération des psychothérapeutes (h/f) free-lance. Grâce à la nouvelle loi, il sera plus facile et plus attrayant pour les autrichiens (h/f) de s'installer en Allemagne sur une base professionnelle. D'autre part, il sera moins attrayant pour les allemands de venir en Suisse, surtout si le modèle de la prescription ne devait pas venir.

Le modèle allemand de mise en place d'un cursus universitaire direct en psychothérapie deviendra un modèle pour d'autres pays européens. Plusieurs dizaines d'universités allemandes (privées et publiques) se préparent à proposer un tel programme ou cursus. Certains instituts de formation postgrade en dehors de l'université, se demandent à juste titre quel sera leur rôle si les universités offrent également une formation postgrade au sein de l'université. Dans ce contexte, il convient également de se référer à l'article «Tendances vers l'académisation de la formation postgrade en psychothérapie » de ce numéro (p. 67) et à la conférence annoncée sur ce thème le 28 mars 2020.

Les travaux du groupe de travail mis en place avec un lobbyiste au Parlement européen pour la reconnaissance de la profession de psychothérapeute $(\mathrm{h} / \mathrm{f})$ en tant que profession indépendante seront poursuivis. Le Parlement européen est réuni à nouveau, c'est donc le bon moment pour parler de psychothérapie et de soins. Le site Web remanié de l'EAP (www.europsyche.org) contient une brochure bien conçue avec une argumentation en faveur de la réglementation de la psychothérapie en tant que profession libérale et d'un meilleur accès à la psychothérapie.

Il y a aussi un nouveau dépliant d'information sur l'EAP. Le groupe de marketing formé il y a quelques années affiche ses premiers résultats. LEAP est également présente sur Facebook avec un groupe accessible au public.

Des efforts sont également déployés pour élever les exigences d'un certificat européen de psychothérapie (ECP) au niveau 7 du cadre européen des certifications $(\mathrm{EQF})$ de manière à ce qu'un
ECP puisse devenir un label pertinent dans toute l'Europe. L'un des problèmes réside toutefois dans le fait qu'aucun État ne reconnaîtra le label d'une association en tant qu'EQF 7, étant donné que ces labels ne sont généralement attribués aux filières de formation continue et de formation postgrade que si un organisme fournissant des formation postgrade du pays concerné organise effectivement une filière de formation postgrade, dispose de salles de formation, etc. Et l'EAP, en tant qu'organisation faîtière, ne le fait pas ellemême. Ce seraient donc les instituts de formation postgrade correspondants appartenant à l'EAP qui devront faire reconnaître leur cursus dans leur pays respectif.

Le Science and Research Committee (SARC) a assisté à deux congrès scientifiques de la Society for Psychological Research (SPR) et a présenté en détail les résultats de l'état actuel de la recherche en psychothérapie. Le conseil d'administration a approuvé l'initiative du SARC de tenir une conférence de recherche à Vienne le dimanche 23 février 2020, à la suite de ces réunions. Lobjectif est d'initier une recherche orientée vers la pratique dans un mélange de méthodes qualitatives et quantitatives, à travers les pays et les méthodes. Le professeur Matthias Desmet, de Belgique, a déjà donné son accord pour présenter son plan de recherche sur la « single case time series " d'une manière orientée vers la pratique et pour présenter des exemples d'études qui ont déjà été réalisées. Le SARC est en train d'inviter un ou deux autres conférenciers $(\mathrm{h} / \mathrm{f})$ qui soccupent de recherche pratique sur les processus et les résultats dans le domaine du «mixed design $»$.

Cette conférence est le résultat d'une mise en réseau au sein de la SPR. Grâce à cette présence, des contacts peuvent être établis avec des chercheurs (h/f) confirmés qui soient alors prêts à explorer les possibilités de coopération avec une organisation de pratique européenne pour la recherche pratique et à s'y impliquer. Le SARC doit insister encore et encore sur ce point lorsqu'il s'agit de défendre le poste budgétaire des indemnités de déplacement et des frais de congrès pour de tels événements.

La base de l'EAP est la Déclaration de Strasbourg sur la psychothérapie. Lannée prochaine, cela fera 30 ans qu'elle a été signée, avec des représentants (h/f) d'Autriche, de Suisse, d'Allemagne, des PaysBas et de la Hongrie. Ce sera l'occasion d'une pe-

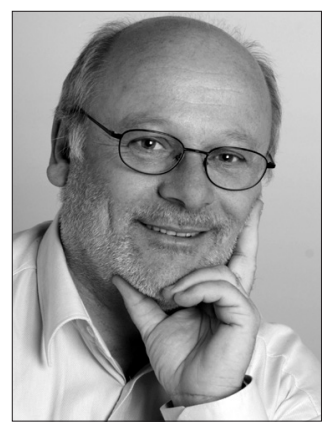


tite célébration. Elle aura lieu le soir du samedi 22 février 2019, après les réunions de l'EAP.

On peut proposer, par exemple, à ceux qui s'intéressent à l'histoire et à la recherche, de passer un week-end à Vienne, ainsi que de participer à l'événement anniversaire et à la conférence de recherche de l'Université privée Sigmund Freud.

À la suite des réunions d'automne à Pristina (Kosovo), le Groupe de travail pour les réfugiés tiendra une conférence sur "L'intégration des réfugiés en Europe : Échange d'expériences dans différents domaines d'activité ». Lobjectif de cette réunion est de donner à tous ceux qui travaillent avec des réfugiés en Europe l'occasion de partager leurs expériences. Elle est également ouverte aux employés d’organismes sans but lucratif. Les principaux organismes d'aide internationale (tels que le HCR et la Croix-Rouge) sont également invités à y participer. La conférence aura lieu les 17 et 18 octobre 2020 .

L'EAP a également complété ses règles d’éthique et a rendu les établissements membres responsables de la protection des étudiants contre les traitements non éthiques, même en cas de difficultés. Jusquà présent, il y avait une lacune dans les directives de déontologie. Les patients $(\mathrm{h} / \mathrm{f})$ étaient protégés, mais les étudiants nétaient pas inclus. Cela est dû à diverses plaintes contre des instituts de formation et des associations professionnelles ou des associations faîtières dans certains pays.

En 2021, se tiendra une prochaine conférence thématique du Comité d'éthique sur les questions éthiques professionnelles dans la pratique.

\section{Peter Schulthess est membre du comité de} l'ASP et représente l'association avec Gabriela Rüttimann dans l'EAP.

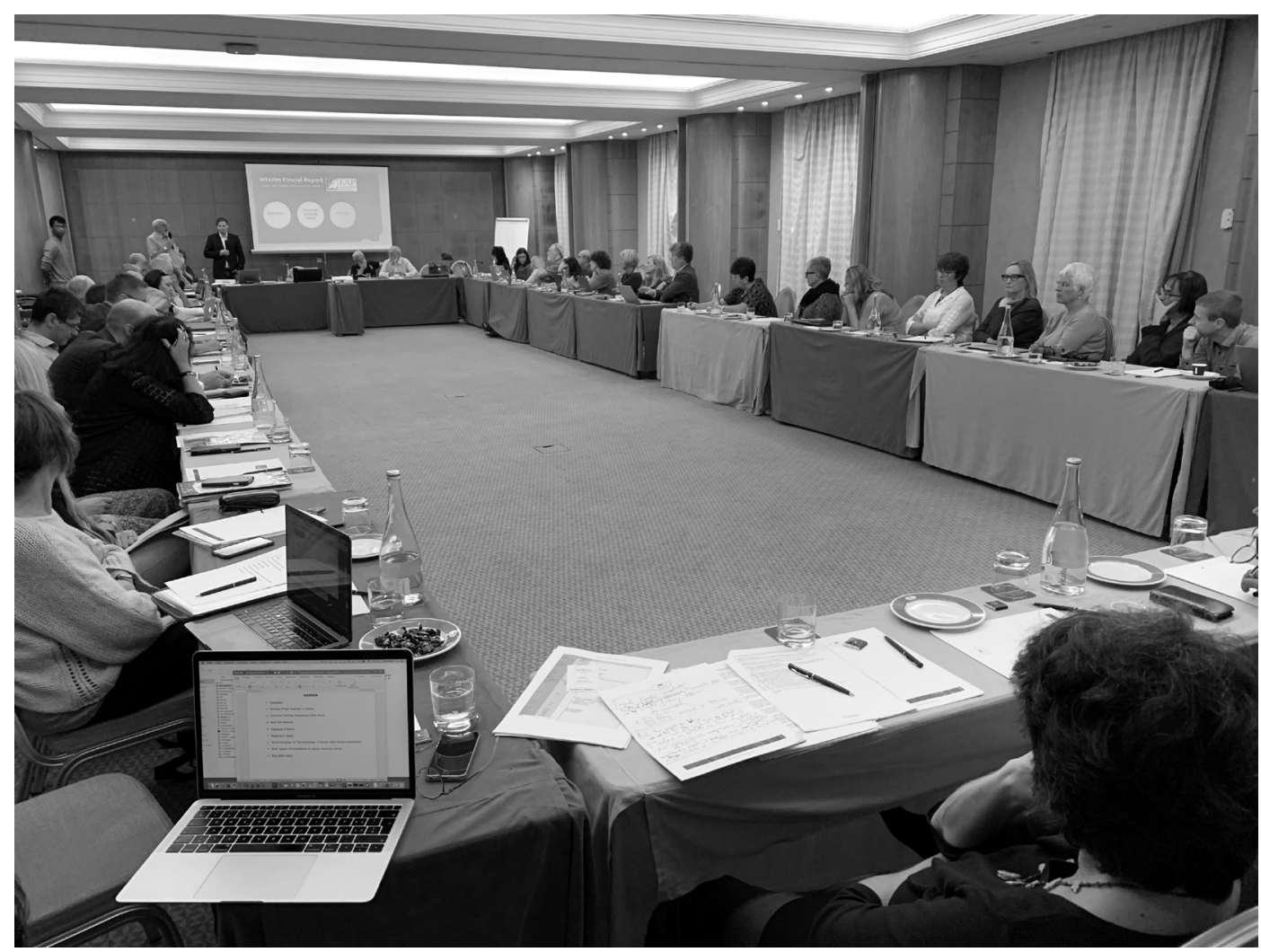

Réunion du Conseil général de l'EAP (Présentation du rapport financier) 